\title{
Le Musée de l'histoire de l'immigration à Paris: une collection et un musée en devenir
}

\begin{abstract}
Andréa Delaplace ${ }^{1}$
RÉSUMÉ: Avec la mondialisation et l'émergence de nouvelles politiques d'immigration dans la Communauté Européenne, la question des migrations est centrale dans le monde politique d'aujourd'hui. C'est dans ce contexte historique de pays d'immigration ou d'émigration que se met en place et s'organise l'étude des musées d'immigration qui appartiennent à la catégorie des musées d'histoire et de société. En France, la Cité nationale de l'Histoire de l'Immigration, qui a ouvert ses portes en octobre 2007, depuis 2013 Musée de l'histoire de l'immigration (MHI), se distingue, dans ce contexte mondial, par l'originalité de son projet et les discussions qu'il suscite dans divers domaines (aussi bien dans le milieu académique que dans les réseaux d'associations qui s'occupent des immigrés en France). Le musée présente au public une approche historique et culturelle de l'immigration ainsi que des œuvres d'art contemporain sur ce sujet. Dans cet article, on s'interrogera sur comment ce musée constitue une tentative de reconnaître le patrimoine de l'immigration comme un patrimoine national.
\end{abstract}

MOTS CLÉS: Immigration. Musée. Identité nationale. Diversité culturelle. France.

RESUMO: Com a globalização e o surgimento da Comunidade Europeia, a questão das migrações se apresenta no centro das preocupações políticas mundiais contemporâneas. E nesse contexto histórico de países de imigração ou de emigração organizam-se os museus de estudos das migrações que pertencem à categoria dos museus de história e de sociedade. Em outubro de 2007, é criada, em Paris, a Cité nationale de l'Histoire de l'Immigration (CNHII) que desde 2013 passou a se chamar Musée de l'histoire de l'immigration (MHI): um museu que apresenta ao público uma abordagem histórica e cultural da imigração assim como obras de arte contemporânea que tratam do tema. Neste artigo, bucaremos compreender como este museu constitui uma tentativa de reconhecimento do patrimônio da imigração como um patrimônio nacional.

PALAVRAS-CHAVE: Imigração. Museu. Identidade nacional. Diversidade cultural. França.

ABSTRACT: Globalization and the emergence of political issues in the European Union have propelled the topic of immigration into the center of the current political climate. In this contemporary
1. Doctorante en Histoire du Patrimoine et des Musées à l'Université Paris 1 Panthéon-Sorbonne, Paris. E-mail: <andreadelaplace@ yahoo.fr>. 
2. Après une longue période de rénovation et restructuration, le Musée de l'homme a rouvert ses portes en octobre 2015.

3. L'ouverture du Mucem a été repoussée à plusieurs reprises jusqu'à son ouverture en juin 2013.

4. Il faut rappeler aussi que le projet du musée de l'immigration a été conçu et lancé sous le gouvernement de Jacques Chirac et que Sarkozy a choisi de ne pas inaugurer ce musée car il ne le voulait pas politiquement (sous le gouvernement Sarkozy les politiques d'immigration ont été très strictes avec la création d'un Ministère de l'Immigration, de l'Intégration, de l'Identité nationale et du Codéveloppement).

5. Dans cet article nous utiliserons le sigle CNHI pour parler du Musée de l'histoire de l'immigration puisque nous parlons de sa création et de son parcours avant les changements récents.

6. Cf. François Hollande (2014).

7. Voir: Immigration: la contre-offensive de Hollande (2014). context, museums that are focused on immigration are gaining more importance due to the impetus for preserving and providing visibility for the heritage of immigrants. In Paris during October 2007 the Cité nationale de l'Histoire de l'Immigration (CNHI) opened its doors but in 2013 it changed its name to Musée de l'histoire de l'immigration (MHI). The museum presents a historical and cultural approach to immigration as well as displaying contemporary works of art that deal with the theme of immigration. In this paper we will analyze how this museum is an attempt to integrate the History of immigration as a national heritage.

KEYWORDS: Immigration. Museum. National identity. Cultural diversity. France.

Dans ce début du XXlème siècle, la France assiste à une redéfinition et à une profonde transformation de son paysage muséal. Avec la création du Musée du Quai Branly, toute une réorganisation des collections d'anthropologie et du système des musées dans ce domaine s'est produite.

Le Musée National des Arts d'Afrique et d'Océanie (MAAO) a fermé ses portes en 2003, ainsi que le Musée National des Arts et Traditions Populaires (ATP) en 2005 et le Musée de l'Homme en 2008². Leurs collections ont été transférées à d'autres musées: respectivement, au Musée du Quai Branly ouvert en 2006, et au MuCEM, à Marseille, finalement inauguré en $2013^{3}$ avec un énorme succès auprès du public.

Dans ce cadre de renouvellement, la Cité nationale de l'histoire de I'immigration (CNHI) ouvre ses portes en octobre 2007, après une longue période de gestation et sans la présence du président de la République de l'époque, Nicolas Sarkozy. De ce fait, son ouverture reste ainsi discrète et sans caractère officiel ${ }^{4}$.

La $\mathrm{CNH}$ est un musée national, un établissement public relevant de la tutelle de trois ministères de l'Éducation Nationale, de la Culture et de la Recherche. Cependant, le musée n'est pas totalement achevé lors de son ouverture et dans les années suivantes d'autres apports vont être ajoutés au projet initial: ouverture de la médiathèque Abdelmalek Sayad, des espaces restaurés du Palais de la Porte Dorée, de l'exposition permanente au deuxième étage sur l'histoire du palais, et création d'un café et d'un espace de convivialité à l'entrée du musée.

Ces deux dernières années ont été marquées par le changement de nom de cette institution, qui devient officiellement Musée de l'histoire de l'immigration en 2013, et par la réouverture au public, en 2014, de l'exposition permanente Repères et de la Galerie des Dons ${ }^{5}$. Avec son inauguration le 15 décembre 2014, sept ans après son ouverture, le musée fait la une des médias et le discours de François Hollande sur l'immigrationº relance les discussions sur cette thématique?

En conséquence, plusieurs questions se posent. La CNHI a-t-elle réussi à mettre en place les objectifs présentés dans son projet scientifique? Quel est le patrimoine de l'immigration et en quoi consiste-til? Comment présenter l'immigration? Quel discours se construit sur l'immigration à travers l'exposition permanente Repères? 
L'objet de cet article est de s'interroger sur les liens entre mémoire, patrimoine, immigration et diversité culturelle. II s'agit de comprendre les enjeux de la création d'un musée national en France consacré à l'immigration. Dans la première partie, on dresse un rappel historique du projet de la CNHI suivi d'une analyse des missions du musée présentées dans son projet scientifique. Dans la deuxième partie, on aborde la question du choix du bâtiment et sa relation avec le musée. Finalement, on analyse la formation de la collection et la constitution d'un patrimoine de l'immigration.

Rappel historique du projet

L'idée d'un lieu consacré à l'histoire de l'immigration était depuis longtemps défendue par les milieux associatifs et universitaires. En 1992, le projet de création d'un musée était au coeur de l'Association pour un Musée de I'Immigration, créée à l'initiative d'historiens et de militants associatifs, notamment de Pierre Milza, Gérard Noiriel, Dominique Schnapper, Emile Temime et Patrick Weil.

En 2001, suite à la demande du Premier Ministre Lionel Jospin, un premier rapport, rédigé par Driss El Yazami, délégué général de l'Association Génériques, et Rémy Schwartz, maître des requêtes au Conseil d'État, réfléchissait sur la forme que pourrait revêtir un lieu dédié à l'histoire de l'immigration. Le rapport prônait la création d'un centre national de l'histoire et des cultures de l'immigration ${ }^{8}$ et avançait plusieurs propositions: un centre national, un réseau de partenaires, un lieu ouvert sur l'université, un musée ouvert au public.

En 2002, le projet d'une cité nationale de l'histoire de l'immigration a été annoncé dans le programme de Jacques Chirac, puis a été relancé dans le cadre plus large du Comité Interministériel d'Intégration du 10 avril 2003. L'une des décisions du Comité concernait la mise en place d'une nouvelle mission, présidée par Jacques Toubon, de préfiguration d'un centre de ressources et de mémoire de l'immigration. Tenant compte des leçons du rapport de El Yazami et Schwartz et se référant aux moyens et aux compétences de l'Agence pour le Développement des Relations Interculturelles (Gip Adri), cette mission a mis en place les outils nécessaires à la réalisation d'une institution à vocation culturelle, sociale et pédagogique nouvelle, destinée à reconnaître et mettre en valeur la place des populations immigrées dans la construction de la France.

En 2004, après un an de travaux et portée par une réflexion universitaire et associative de plusieurs années, le projet de la Cité Nationale de l'Histoire de I'Immigration a été officiellement lancé le 8 juillet 2004. L'établissement public de la Porte Dorée9, Cité Nationale de l'Histoire de l'Immigration, est créé le ler janvier 2007, après parution au Journal Officiel du 17 novembre 2006. L'ouverture du musée est annoncée pour le printemps, puis l'été 2007 et, finalement, l'ouverture aura lieu le 10 octobre 2007.
8. Voir GENERIQUES (2015).

9. Le Palais de la Porte Dorée, établissement culturel public de l'Etat, abrite deux projets scientifiques et culturels différents : la Cité nationale de l'histoire de l'immigration - aujourd'hui Musée de l'Histoire de l'immigration - et l'Aquarium tropical vestige de l'Exposition coloniale de 1931. 
10. Cf. CNHI, Projet scientifique et culturel (2005).

11. Cf. Marie-Hélène Joly (2007, p. 68).
Missions

Dans son projet scientifique et culturel, la CNHI révèle sa mission et son caractère de musée. Des quatre grands objectifs avancés par les concepteurs du projet, les deux premiers montrent très clairement l'attachement de la $\mathrm{CNHI}$ à la question du patrimoine.

1. "Concevoir et gérer le musée national de l'histoire et des cultures de l'immigration, ensemble culturel original à caractère muséologique et scientifique, chargé de conserver et de présenter au public des collections représentatives de l'histoire, des arts et des cultures de l'immigration."

2. "Conserver, protéger et restaurer pour le compte de l'État les biens culturels inscrits sur l'inventaire du musée national de l'histoire et des cultures de l'immigration dont il a la garde et contribuer à l'enrichissement des collections nationales"10.

Ainsi la CNHI se définit avant tout, comme un musée national de l'histoire et des cultures de l'immigration, chargé de conserver, protéger et enrichir des collections et de les diffuser auprès du public. Le choix du lexique utilisé ici est essentiel pour comprendre la volonté de rattacher la $\mathrm{CNHI}$ à la question patrimoniale. Selon Marie-Hélène Joly:

"La CNHI aurait pu ne pas être un musée: baptisée" Centre de ressources et de mémoire " dans le rapport de la mission de préfiguration de 2004, son nom définitif a fait l'objet de plusieurs hypothèses, chacune révélant des intentions et un positionnement symbolique différents. Cependant, sa mission de musée a été clairement inscrite dans tous les textes de création de l'institution certes en partie pour des raisons conjoncturelles de recherche de financement, mais il n'est pas indifférent que les associations à la base du projet aient souhaité cette inscription, révélant par là un attachement à la nature patrimoniale et pérenne de l'institution"musée". L'existence d'un patrimoine leste en effet un établissement: le patrimoine témoigne visiblement de l'existence d'un phénomène et par ailleurs on ne peut faire disparaître d'un trait de plume un monument, une collection"11.

Le patrimoine reste ainsi une façon de sauvegarder la mémoire de l'immigration qui renforce son caractère pérenne par la création d'une collection ef d'un musée. Si le projet de la CNHI avait été d'un centre de ressources et de mémoire, comme le rappelle Marie-Hélène Joly, le poids et la portée de l'institution n'auraient pas été les mêmes.

Cette mise en patrimoine de l'immigration repose donc sur un objectif d'agir sur la société et ses représentations. Autrement dit, la reconnaissance patrimoniale et ses outils peuvent engendrer un changement de la réalité en passant par un changement des représentations d'une culture ou d'une communauté. Ainsi, le musée national de l'histoire et des cultures de l'immigration affiche dans son nom cette volonté de reconnaissance de la diversité culturelle liée à l'immigration.

Pourtant, avec le changement du logotype de la CNHI en 2013, cette mention à la diversité culturelle disparait. La CNHI devient alors Musée de l'histoire de l'immigration. Ce changement a eu lieu avec une nouvelle campagne de 
communication qui mettait l'accent sur la mise en patrimoine de la mémoire de l'immigration. Sur le site internet du musée on peut lire: "À travers des messages qui interpellent le grand public, avec une accroche simple, directe et humoristique, cette campagne entend rappeler que l'histoire de l'immigration est l'histoire de tous, et fait partie de l'histoire de la France" 12 .

Toutefois, cette mémoire ne peut évidemment être univoque, tant les mobilisations associatives ou autres divergent. Telle association va afficher son souhait de rassemblement des immigrés de toute origine, telle autre ne concevra sa vocation mémorielle et patrimoniale que dans la singularité de l'histoire d'une communauté en particulier. En conséquence, la question de la diversité culturelle face au patrimoine reste toujours complexe: volonté multiculturelle versus volonté communautaire. Quand on parle de questions mémorielles c'est plus facile de parler de la mémoire d'un groupe de personnes, d'une culture. On peut se demander s'il n'y a pas une certaine artificialité à vouloir parler de mémoire ou de patrimoine de I'immigration, qui suppose un discours basé sur la conception d'Etat-nation, alors que les mobilités de populations obéissent finalement à des logiques transnationales, mais aussi une identification collective à une situation migratoire qui regroupe des réalités extrêmement diverses. De ce fait, est-ce que les populations immigrés peuvent-elles ou, encore, veulent-elles se reconnaitre dans une même histoire, un même patrimoine comme le propose la $\mathrm{CNHI}$, née de la volonté de donner une "place aux immigrés" dans le grand récit national?

Le processus de patrimonialisation de l'immigration reste ainsi très délicat justement par le caractère multiculturel de ce phénomène qui va à l'encontre de l'unité du discours patrimonial. Néanmoins, même si la Cité traite d'un thème complexe à gérer, comme celui de l'immigration, son ambition et sa portée restent nouvelles car, avant la création de la CNHI, il n'y avait pas de musée destiné à I'histoire de l'immigration en France. À l'exception de quelques expositions temporaires ou expériences dans quelques musées, le thème était absent du panorama des musées français:

Ce n'est pas un vide absolu, néanmoins: quelques musées ont travaillé avec les communautés immigrées, mais ils sont peu nombreux. On peut citer l'Écomusée de Fresnes, le Musée Dauphinois de Grenoble et le Musée d'Histoire de Marseille qui se sont fortement engagés dans cette voie et il faut saluer leur engagement profond et régulier sur cette question ainsi que leur collaboration réelle et respectueuse avec les communautés ${ }^{13}$

Le caractère politique et social de la Cité est très présent dans tous les documents et publications officiels du musée. La volonté d'inscrire l'histoire de l'immigration dans l'Histoire de France est l'un des propos forts, avancés par les porteurs du projet du musée et ainsi, le projet de la Cité s'affirme comme un acte politique vers "I'intégration" de l'histoire et des cultures de l'immigration dans l'histoire nationale. Selon Jacques Toubon, président du Comité d'Orientation de la CNHI, le projet de la Cité est $d^{\prime \prime \prime}$ inscrire l'histoire de l'immigration non seulement comme une composante de I'histoire sociale, mais comme une partie intégrante de l'Histoire de France ${ }^{\prime \prime 14}$. 
15. Cf. CNHI. Dossier de presse (2007).

16. Cf. Marie-Hélène Joly (2007a, p. 75).

17. Voir Benoit de l'Estoile (2007, p.14).

18. Cf. Nöel Barbe et Marina Chauliac (2014, p. 5).
En outre, selon le dossier de presse de la CNHI du printemps 2007:

La Cité veut être un élément majeur de la cohésion sociale et républicaine de la France. Audelà de sa fonction patrimoniale, elle a aussi un rôle important de producteur de culture et de signes. Ses missions principales sont donc des missions au long cours, dont les enjeux fondamentaux se joueront sur plusieurs années ${ }^{15}$.

Ici, on aperçoit donc, de manière très claire, le caractère national et républicain de la CNHI. L'intégration de l'histoire de l'immigration à l'histoire nationale est l'objectif majeur de cette nouvelle institution. Le projet réaffirme donc les concepts d'intégration et de cohésion sociale et républicaine:

[...] Le projet politique qui fonde la $\mathrm{CNH}$ est celui de l'intégration républicaine, appuyé sur une vision historique de longue durée, deux siècles de l'histoire nationale. On peut se demander s'il ne vient pas un peu tard, alors même que cet idéal d'intégration peut paraître aujourd'hui fragilisé ou du moins questionné, mais il a le mérite de relever clairement le défilo.

Sans vouloir trop simplifier, on pourrait résumer l'ambition de ce projet comme étant celui de montrer la façon dont les "Autres", les étrangers immigrés, intègrent progressivement l'identité nationale, le "Nous" nationall". Or on retrouve le même problème qui a été évoqué précédemment: l'approche régalienne du patrimoine en France se heurte à différents obstacles quand il s'agit de l'immigration dont le premier réside dans la conception intégratrice de l'identité nationale française qui va à l'encontre de l'idée de diversité culturelle au sein de la société:

L'apparition d'un patrimoine en lien avec la construction de la nation française laisse peu de place au patrimoine de l'immigration, que celui-ci concerne un patrimoine culturel, lié à I'histoire particulière d'un groupe, ou un patrimoine en lien avec le parcours migratoire : tous les deux renvoient à la fois à la peur du communautarisme et à un imaginaire postcolonial empreint de stéréotypes ${ }^{18}$

En conséquence, on peut dire que la $\mathrm{CNH}$ l possède un projet "à la française" basé sur une vision intégratrice et nationale de l'immigration. Dans des musées anglo-saxons, par exemple, la notion de communauté semble être plus présente dans le processus de patrimonialisation et cela vient peut-être du fait que, dans ces pays, l'état est moins présent dans les initiatives de mise en patrimoine. Parallèlement, c'est la première fois depuis de nombreuses décennies que l'État français s'engage dans un projet de musée national à caractère social et politique si fort, comme celui de la CNHI.

Le choix du bâtiment

Plusieurs lieux ont été envisagés pour abriter la Cité Nationale de I'Histoire de l'Immigration: la Bourse de Commerce, une partie du Palais de Chaillot, I'Hôpital Laennec, l'ancien Centre Américain à Bercy ou même, le toit de la Grande Arche. Finalement, le choix du bâtiment s'est porté sur le Palais de la Porte Dorée 
"en raison de son statut de monument historique, de son rayonnement artistique et de son style architectural spectaculaire et limpide, trois éléments qui en font un lieu prestigieux. Par ailleurs, le rôle de ce palais dans l'histoire de France, en l'occurrence I'Exposition Coloniale Internationale de 1931, invite à revisiter l'histoire des liens entre la France et le reste du monde et l'évolution du regard qu'elle porte sur les autres cultures"19.

Ce choix a été polémique car le Palais de la Porte Dorée fut le siège de I'ancien Musée des Colonies inauguré lors de l'Exposition Coloniale de 1931. Ainsi, le "poids historique" du lieu alimentait la crainte que le thème de l'immigration en France ne soit mêlé à celui de la colonisation, donc de manière stigmatisante pour le nouveau musée. Pourtant, les porteurs du projet de la $\mathrm{CNHI}$ défendaient qu'il s'agissait de renverser les significations du bâtiment: "d'un lieu de mémoire, d'une forme de glorification de la mission civilisatrice de la France dans les colonies, il doit devenir l'institution culturelle qui illustrera l'apport décisif des immigrés dans la construction et l'identité du pays" 20 . Cependant, ce pari est-il atteint? La Cité a-telle réussi à transformer "l'imaginaire" lié au Palais de la Porte Dorée?

L'histoire du Palais de la Porte Dorée

Le Palais de la Porte Dorée a été construit en 1931 par Albert Laprade et inauguré lors de l'Exposition Coloniale. II a été le seul édifice destiné à survivre à l'Exposition Coloniale car il fut conçu dans le but de rendre pérenne le discours colonialiste.

Le palais, en style art déco comme nous pouvons voir sur la photo de son entrée monumentale (Figura 1), a été conçu afin de cristalliser la diversité des styles de l'Empire et exprimer la domination de celui-ci sur ses colonies. Son plan carré, parfaitement symétrique, reprend le principe des palais marocains en aménageant une grande salle centrale entourée de galeries. Cependant, la façade renove avec la monumentalité des temples antiques, avec des piliers aux chapiteaux stylisés qui évoquent les colonnes ioniques des temples grecs, tout en exprimant la domination de la France sur les colonies représentées par les bas-reliefs. La décoration intérieure du bâtiment, murs et sol, compte plusieurs fresques et mosaïques décoratives, comme nous pouvons voir sur le visuel 2 (Figura 2), qui ont été restaurées et présentées au public pendant les travaux de réaménagement du palais.

Le Palais de la Porte Dorée a changé plusieurs fois de nom, de "Musée Permanent des Colonies" pendant l'exposition Coloniale de 1931, il prend le nom de "Musée des Colonies et de la France Extérieure" en 1932, puis celui de "Musée de la France d'Outre-Mer" en 1935.

Avec la décolonisation, le musée change de vocation et dès 1959, sous I'impulsion d'André Malraux, ministre d'état chargé des affaires culturelles, il devient, en 1960, le "Musée des Arts Africains et Océaniens" (MAAO) fermé au public en 2003, ses collections étant alors transférées au musée du quai Branly.
19. Cf. CNHI. Projet scientifique et culturel de la Cité nationale de l'histoire de l'immigration (2005).

20. Cf. Jean-Luc Marty (2008). 


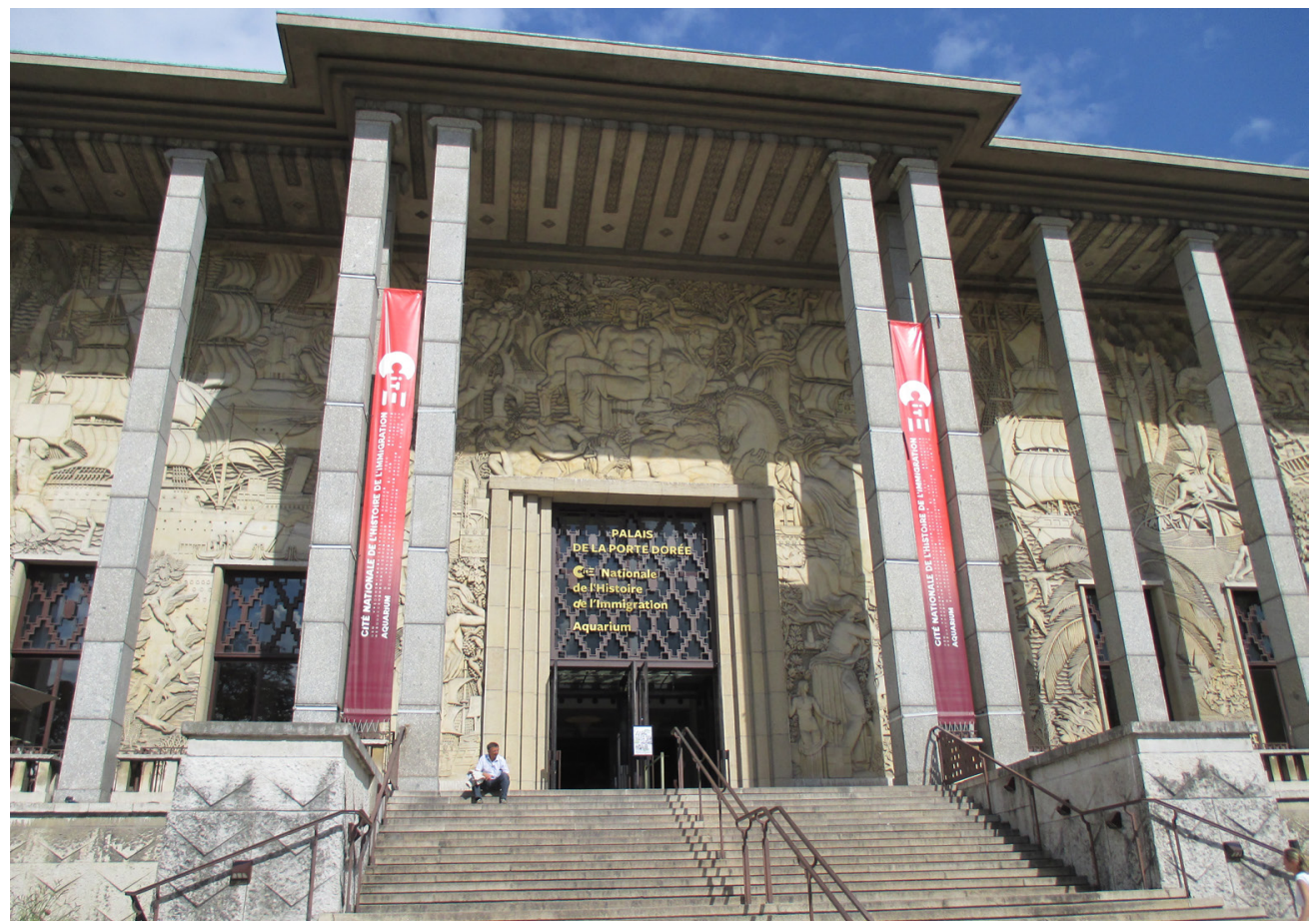

Figura 1 - Entrée du Palais de la Porte Dorée, construit pour l'exposition coloniale de 1931 et qui accueille aujourd'hui le Musée national de l'histoire de l'immigration. Photographie de l'auteur, 2015.

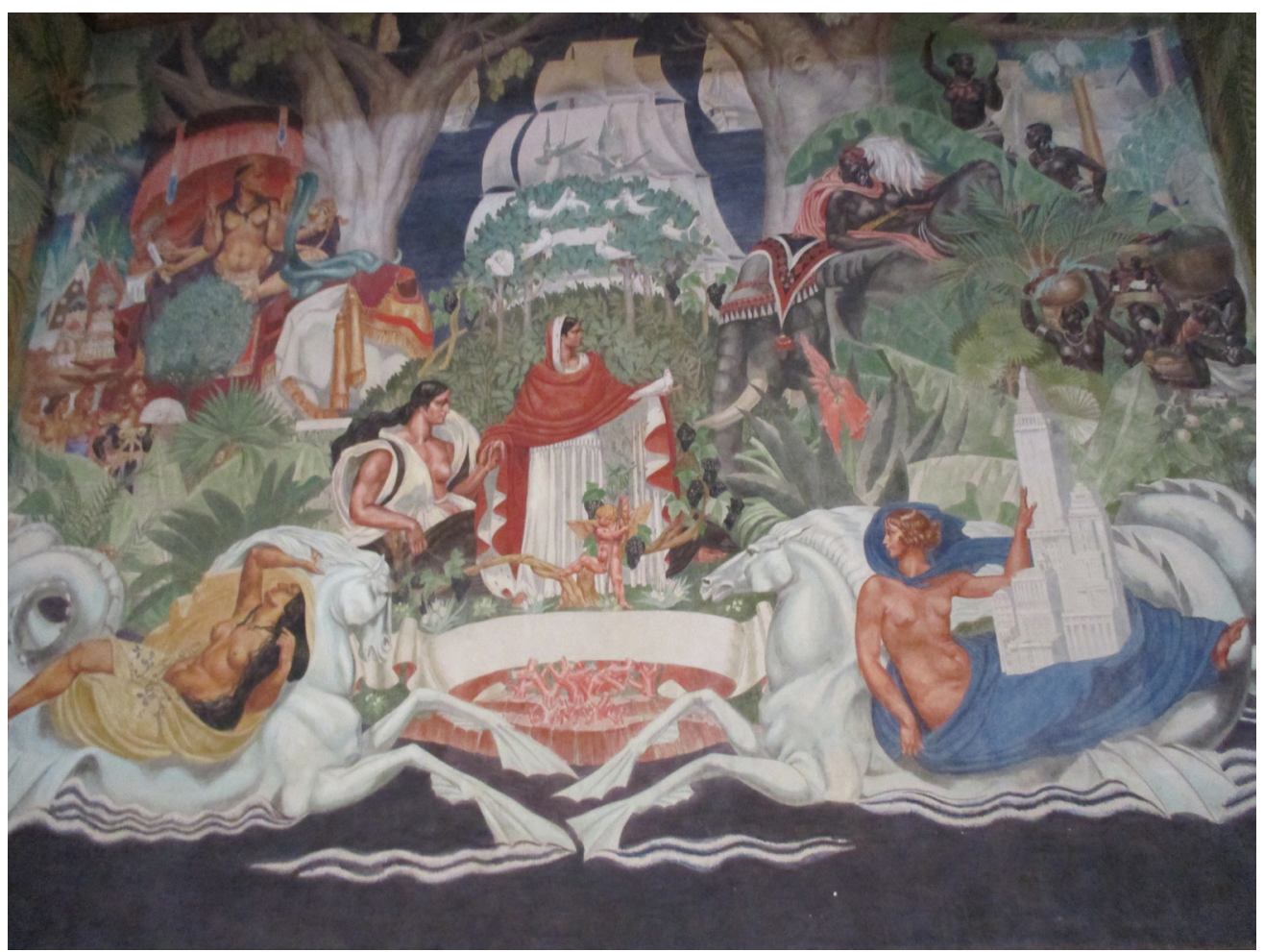

Figura 2 - Salle des fêtes: Mosaïque datant de l'époque coloniale. Photographie de l'auteur, 2015. 
La CNHI ferme symboliquement l'ancien Palais des Colonies le 14 juillet 2006, en programmant une représentation du "Discours sur le colonialisme" d'Aimé Césaire, avant les travaux qui lui permettront d'ouvrir ses portes au public. Cet événement réaffirmait ainsi la nouvelle vocation du lieu: d'un palais des colonies il se transformait en une cité dédiée à l'histoire et aux cultures de l'immigration. Selon les porteurs du projet de la CNHI, le bâtiment devenait un lieu vivant, un vrai forum de rencontres et de discussions à propos de l'immigration.

Pourtant, quand on visite le musée, la réalité se révèle complètement différente du projet. Le Forum central, ancienne salle des fêtes, où l'impression de vide est très marquée, en est un exemple manifeste. On se déplace dans la grande salle des fêtes sans trop savoir quel est le but de cette salle. C'est un énorme espace avec un très haut plafond, où l'on peut se déplacer à sa guise mais sans comprendre le contexte des mosaïques et fresques qui décorent la salle. Les visites guidées du musée commencent par la salle des fêtes pour mettre le bâtiment dans son contexte et présenter son histoire. Cependant, le visiteur qui choisit de faire, seul, la visite, sans audio-guide ou visite guidée, ne rencontre pas de support écrit (note ou texte de présentation) ni de mise en contexte du décor de la salle des fêtes, à l'entrée du musée.

Le premier étage, qui n'avait aucune exposition permanente (à l'exception d'une maquette du Palais de la Porte Dorée et de quelques expositions temporaires), présente depuis 2013 une exposition sur l'histoire du Palais et sa mise en contexte qui est une réponse aux critiques faites à ce sujet lors de son ouverture en 2007. II faut monter au deuxième étage du musée pour accéder à l'exposition permanente Repères, à l'espace destiné aux expositions temporaires et à la Galerie des dons.

De plus, la CNHI n'occupe qu'une partie du Palais de la Porte Dorée, le sous-sol étant occupé par l'Aquarium. Les salles, qui ne font pas partie des espaces d'exposition, restent inoccupées et on a l'impression que le musée ne s'approprie pas vraiment de son espace physique. On ressent un énorme décalage entre ce qui est exposé et le bâtiment.

D'un côté, on retrouve l'exposition permanente Repères dans un espace réaménagé qui possède une muséographie très contemporaine. De l'autre côté, pour les espaces restaurés pendant les travaux du musée, comme les fresques de la salle des fêtes ou le salon Lyautey, on ne dispose que de très peu d'informations à l'entrée des salles. Depuis 201 1, quelques dépliants disponibles, à l'accueil du musée, tentent de combler cette lacune. Il est clair qu'il faut procéder à une mise en contexte plus effective des espaces historiques du Palais.

II serait intéressant d'intégrer espace muséal et bâtiment pour que le public puisse mieux appréhender la décoration et l'espace historique dans lequel la CNH s'est installée. Même si depuis 2013, l'exposition du 2ème étage essaie de faire cette mise en contexte essentielle du Palais de la Porte Dorée, elle reste une réponse tardive et insuffisante.

La CNHI semble craindre de mélanger histoire de l'immigration et histoire coloniale et, de ce fait, laisse de côté une mise en contexte permanente ${ }^{21}$ de la
21. Pendant l'exposition temporaire 1931, les étrangers au temps de l'Exposition Coloniale, en 2008, la relation entre 1'Exposition coloniale, le Palais de la Porte Dorée et l'immigration a été très bien analysée. 
22. Cf. Marie-Hélène Joly (2007, p. 68-69). relation entre le bâtiment qu'elle occupe et l'histoire coloniale. Pourtant, cette ouverture à la discussion de l'histoire coloniale est très importante, même si ce n'est pas l'objet principal du musée. Le bâtiment qui abrite la cité est trop chargé d'histoire pour que celle-ci soit oubliée. La sensation de décalage entre expositions et bâtiment que ressent le visiteur mérite d’être allégée.

Le défi de la constitution d'une collection

Un comité scientifique pluridisciplinaire, qui regroupe des professionnels issus de formations très diverses comme l'histoire, I'histoire de l'art, l'art contemporain, I'art du XIXème siècle, I'histoire de la photographie, l'ethnologie et l'anthropologie sociale; a été chargé d'élaborer un plan d'action pour le musée. Parmi ces professionnels qui ont participé à la création du musée, on trouve: Nancy Green, Gérard Noiriel, Marie-Claude Blanc-Chaléard et Patrick Weil. Les séances de travail ont été l'occasion de larges débats où savoirs et expériences ont été échangés afin d'opérer des choix pertinents aussi indiscutables que possible. Cependant, selon ses réalisateurs, la contrainte d'un calendrier serré, - les galeries permanentes étant construites simultanément à la constitution des collections -, a été une difficulté majeure du projet.

En conséquence, la $\mathrm{CNH}$, en un court espace de temps, a dû relever le défi de commencer une collection à partir de rien, car elle n'avait hérité d'aucune collection initiale. Le défi de la page blanche a été l'un des points forts lors des discussions du comité scientifique. Comme le rappelle bien Marie-Hélène Joly:

Tous les musées nationaux sont nés de collections : confiscations révolutionnaires, libéralités et dations prestigieuses, commandes de l'État appelées avec le temps à accéder au statut de musée, recompositions de collections existantes. L'histoire des musées nationaux sur deux siècles est faite de ces recompositions et migrations de collections, y compris très récemment lainsi le musée national d'art moderne, le musée d'Orsay, le musée du Quai Branly, le MuCEM²2.

Désormais, ce défi de création d'une collection reste une première dans la création d'un musée national en France. La définition d'un patrimoine de l'immigration constituait une interrogation centrale de ces discussions qui devait permettre de dégager les axes principaux de la constitution des collections. En quoi consiste un patrimoine de l'immigration? Quels objets ou documents doiton privilégier? L'historien travaille principalement avec des documents d'archives, de l'écrit. Alors comment exposer cela dans un musée sans rendre l'exposition ennuyeuse? L'idée d'introduire des œuvres d'art contemporaines pour diversifier les sources s'unit à la volonté de rendre l'exposition plus attrayante et intéressante pour le public.

C'est ainsi que dans la première partie de l'exposition permanente Repères, on peut voir des boites en verre, initialement colorées mais depuis 2014 transparentes comme on peut observer sur la photo 3 (Figura 3), qui contiennent des objets divers: valise, châle, statuette, vinyle, photos, etc. Ce sont des objets qui illustrent différents parcours et histoires de migrants originaires de plusieurs parties du monde. 


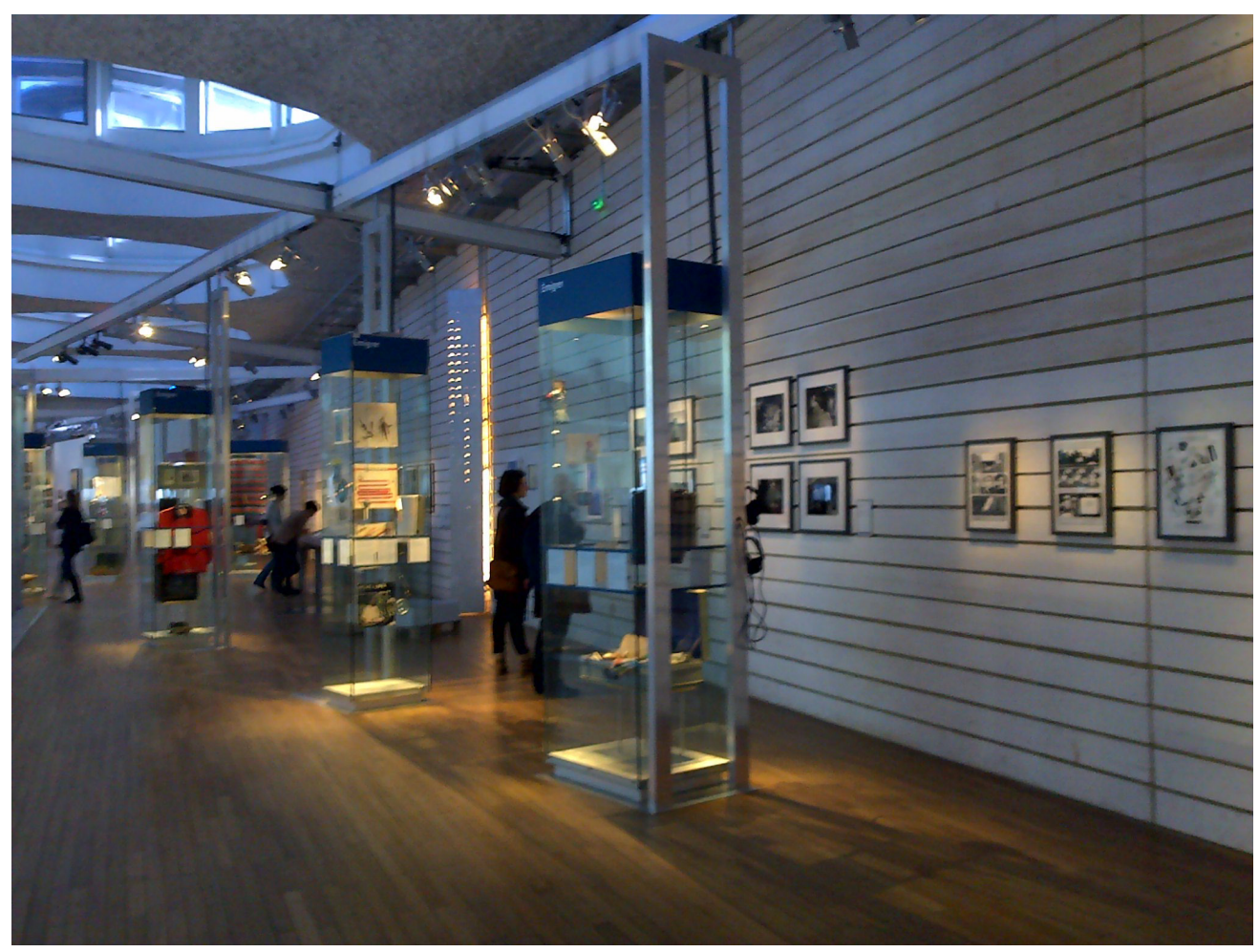

23. Voir d'Arjun Appadurai (1986).

24. Cf. Octave Debary et Laurier Turgeon (2007, p.3).

25. Cf. Octave Debary et Laurier Turgeon (2007, p.5).

Figura 3: Vue de l'exposition permanente Repères. Photographie de l'auteur, 2015.

Ces objets se présentent alors comme étant des objets témoins dépositaires de parcours de vie, porteurs concrets de témoignages et de souvenirs. L'objet devient ainsi détenteur d'une histoire et d'une mémoire unique qui font partie de sa "biographie"23. \|l apporte des réminiscences ou rappelle des souvenirs à celui qui le possède : la mémoire de son origine, de sa famille, de la vie qu'il a laissée derrière lui:

En effet, les objets domestiques de la vie quotidienne réactivent à chaque instant de la journée les souvenirs des personnes et des événements, et les situent dans le registre mémoriel. II s'agit d'une mémoire non verbale qui fait appel aux sens, surtout à la vue, au toucher et à l'odorat ${ }^{24}$.

L'objet prend ainsi le rôle d'un " aide-mémoire ", un support mnémonique qui sert à rappeler des lieux, des personnes ou des évènements significatifs. Néanmoins, les objets matériels, ainsi que les lieux, ne font pas juste nourrir ou activer la mémoire, ils participent à sa structuration. Laurent Lepaludier rappelle que: "I'objet est non seulement une référence cognitive qui cristallise autour de lui la perception du monde, mais aussi un point d'accroche essentiel de la mémoire qui structure le souvenir autour de lui"25.

Le rapport entre objets, lieux et mémoire devient une relation qui se transforme avec le temps et varie selon les personnes. Un même objet peut donc apporter différents souvenirs selon les transformations de la mémoire au long des années. Le tri d'objets pendant un déménagement, par exemple, conduit à un tri de la mémoire et en 
26. Cf. Octave Debary et Laurier Turgeon (2007, p.7). conséquence à une restructuration de celle-ci. La personne doit choisir entre abandonner ou non des objets avec ses souvenirs. Les objets conservés sont requalifiés et la mémoire reconstruite. "Ces objets conservés acquièrent une plus-value mémorielle justement parce qu'ils accumulent des histoires. Leurs riches biographies attestent de la survivance aux drames répétés de la confrontation de l'objet avec ses souvenirs" 26 .

Ainsi, "la mémoire des lieux ou des objets n'est pas figée, elle est appropriée et domestiquée de toutes sortes de manières par les citoyens". La mémoire d'un monument, par exemple, peut changer "au cours de la vie des gens en fonction de l'évolution des contextes ou des besoins des personnes elles-mêmes." C'est ce qui se passe avec le Palais de la Porte Dorée qui veut transformer son rôle dans la mémoire collective des français d'un lieu lié à la colonisation vers un lieu lié à l'immigration.

Cependant, l'héritage des migrants ne se constitue pas seulement d'objets mais aussi de témoignages et de mémoires immatérielles. En effet, l'héritage culturel des immigrés se compose en grande partie d'un héritage immatériel (mémoires, réminiscences) et les objets demeurent minoritaires. Ainsi, le témoignage de leurs détenteurs devient alors absolument indispensable, qu'il soit direct - témoignage filmé du vivant de la personne -, ou indirect - témoignage laissé par écrit ou transmis oralement. Il est fondamental de laisser s'exprimer la mémoire, réalité d'un groupe ou d'un individu, et ce, quel que soit son rapport à la vérité de l'Histoire, à laquelle elle est en permanence confrontée.

La CNHI a donc décidé de constituer une collection en constante évolution qui unit la collecte d'objets à la collecte de témoignages: c'est la Galerie des dons. Celle-ci reçoit la collecte des objets du quotidien d'hier, devenus souvenirs et peut-être symboles pour ceux qui les possèdent. Le visiteur peut contribuer à cette collection en faisant un don, un prêt ou un dépôt. Afin de faire partager ces histoires, d'en montrer la richesse et la diversité, d'en faire un patrimoine commun, chaque don sera accompagné d'un récit, d'une explication. L'objet "témoin" est alors accompagné d'un récit - héritage immatériel - qui l'enrichit.

Alors, en quoi consiste le patrimoine de l'immigration selon la $\mathrm{CNHI}$ ? Si on analyse la constitution de la collection du musée, on s'aperçoit qu'elle repose sur une conception mémorielle du patrimoine. Le parcours personnel de l'immigré est valorisé ainsi que l'histoire orale. La notion d'objet témoin reste centrale dans la constitution de cette collection ainsi que la pluralité de ces objets qui témoignent de la diversité culturelle des communautés d'origine.

Ce sont les objets témoins et les documents personnels - tels que photos, documents officiels, pièces d'identités, passeports - donnés par les immigrés, qui permettent de retracer le parcours de vie de leurs propriétaires. De ce fait, l'enjeu que représente la définition d'un patrimoine de l'immigration semble indissociable d'un travail de collecte d'objets et de récits auprès des populations d'immigrés de proche ou lointaine descendance. Cette stratégie de constitution de la collection aboutit ainsi à une approche très mémorielle de l'immigration dans la Galerie des dons et dans la première partie de l'exposition permanente Repères, ratifiant le lien entre mémoire, immigration et diversité culturelle. 
L'axe central de l'exposition permanente consiste en une analyse de I'histoire de l'immigration en France sur une période donnée, du début du XIXème siècle jusqu'à aujourd'hui. Selon ses réalisateurs, l'idée était de "porter un œil nouveau sur l'histoire de France et montrer la part prise par les immigrés dans le développement économique, les évolutions sociales et la vie culturelle de la France" 27 . L'initiative est d'engager le public au rythme d'un parcours historique et "émotionnel" relatant des temps forts de l'histoire de l'immigration en France.

D'un point de vue méthodologique, l'idée est d'en définir les moments forts, les phases de rupture, les longs cheminements et la pluralité des regards de communautés se situant dans l'accueil ou dans l'exil. Ainsi à partir de cet axe central, plusieurs questions ont surgi: "Comment rendre séduisant le discours complexe et sensible d'une histoire souvent douloureuse? Quelles collections constituer afin de présenter cette histoire dans une exposition permanente?"28.

La construction d'un discours spécifique au musée est, donc, essentielle lors de la création d'une exposition. Celle-ci est un discours qui se compose de documents, d'images, d'objets, d'œuvres et de textes, incontournables pour la compréhension du sujet traité. Comme le musée ne possédait pas une collection déjà constituée, la création du discours a été, cependant, un peu plus libre. "En, effet, les collections existantes ne permettent pas toujours de développer un discours pertinent et cohérent, les traces ne sont pas forcément significatives, le stock peut parfois se révéler plus encombrant que véritablement utile et induire une pesanteur voire un frein dans la réflexion. [...] L'absence de collections laisse au contraire la voie libre à la réflexion et permet d'élaborer un véritable projet intellectuel"29.

Ainsi, grâce au manque de collections préexistantes ${ }^{30}$, le comité scientifique, en charge de l'exposition permanente, a eu une autonomie plus grande de choix pour élaborer un discours sur l'immigration. Un parcours thématique a, donc, été préféré à une approche chronologique ou par nationalité. L'idée était de créer un parcours qui retracerait la trajectoire d'un immigré (départ, terre d'accueil, logement, travail...) en abordant, différents thèmes liés à l'histoire de l'immigration dans chaque partie de l'exposition. D'autres questions ont surgi. Comment aborder chacune de ces thématiques ? Quelle approche fautil privilégier? Le choix d'un croisement des regards, présent dès le rapport de la Mission de préfiguration du musée, s'est révélé indispensable pour permettre au visiteur de saisir les différents aspects d'une même thématique. Les approches historique, anthropologique et artistique sont, désormais, préférées. Même si la dimension historique est privilégiée, - car c'est un musée d'histoire -, l'apport des autres disciplines est essentiel lors de la construction de l'exposition. Ces regards, différents mais complémentaires, ouvrent la voie à une compréhension plus profonde du sujet, sans imposer de vérités et sans exclure de nouveaux thèmes. Ainsi, l'idée est de traiter les thématiques liées à l'immigration par le biais d'un discours multidisciplinaire pour que le visiteur puisse, par conséquent, être en situation de questionnement, voire de remise en cause de ses propres représentations sur le phénomène migratoire.
27. Cf. CNHI, Dossier de presse (2007).

28. Cf. Hélène LafontCouturier (2007).

29. Cf. Marie-Hélène Joly (2007, p. 70).

30. Le défi de la page blanche, évoqué plus tôt dans cet article, a aussi permis une liberté plus grande de création du discours central de l'exposition permanente puisque les collections ont été créés pour illustrer ce discours. 
31. Cf. Hélène LafontCouturier (2007).
Chaque discipline met une lumière différente sur le thème de l'immigration. Le regard historique, basé sur le fait retrouvé dans les archives pour remonter le cours du temps jusqu'au présent, permet une mise en contexte de deux cents ans d'immigration en France. L'historien apporte un regard plus objectif sur I'histoire de l'immigration; un regard basé sur des documents historiques et objets témoins.

En revanche, le regard anthropologique s'appuie principalement sur des faits contemporains observés permettant de faire un retour vers le passé et d'analyser, à partir de la parole des immigrés, les évolutions de l'identité concernée. L'anthropologue porte un regard contemporain sur les communautés immigrées, en recueillant des témoignages et en y alliant une analyse des objets collectés ou donnés.

Le regard artistique, quant à lui, propose une interprétation esthétique subjective - voire émotionnelle - du fait migratoire et offre, ainsi, une autre piste de lecture au visiteur. L'artiste souvent apporte une vision plus sensible qui incite le visiteur à percevoir les autres dimensions d'une même question. II enrichit la discussion de l'immigration en ouvrant une sphère sentimentale qui fait appel à la subjectivité plus qu'à l'objectivité des objets témoins ou documents exposés.

Dans chaque partie de l'exposition permanente on retrouvera, en conséquence, les regards de l'historien, de l'anthropologue et de l'artiste, à travers le choix de documents historiques qui dialoguent avec des objets témoins et des œuvres d'art. Ainsi la politique d'acquisition du musée s'oriente vers les documents historiques, les objets témoins et les œeuvres d'art contemporaines. Actuellement, les collections sont structurées en deux grands pôles, l'un consacré aux œuvres artistiques, l'autre aux parcours et lieux de vie des immigrés.

La place de l'image dans la collection - La collection photographique

L'image a une place centrale dans la collection du musée, soit comme document historique soit comme œuvre d'art. La photographie est le support privilégié de l'image choisi par les conservateurs des collections du musée du fait de sa capacité de documentation et son pouvoir d'attraction sur le visiteur. Selon Hélène Lafont-Couturier, "une photographie relève à la fois d'un extrême de certitude (elle atteste une situation qui a existé) et d'un extrême d'incertitude (nous n'étions pas témoin de ce qui a rendu possible l'image)" ${ }^{\prime \prime}$. L'image a ainsi un très fort pouvoir d'attirance sur les visiteurs.

La collection photographique du Musée de l'histoire de l'immigration se répartit en trois volets et axes d'acquisitions:

- Collection historique - photographies historiques, cartes postales, photographies anciennes.

- Collection d'art contemporain - photographie artistique.

- Collection ethnographique - albums de famille, photos d'archives personnels données par des immigrés (en lien avec leur récit personnel). 
Le travail de prospection iconographique a commencé en 2005 lors de la création d'un documentaire ${ }^{32}$ sur l'histoire de l'immigration en France, mis en ligne sur le site officiel en juillet 2006 avant l'ouverture du musée. Ce film est composé de 350 photographies et de documents d'archive, ponctué d'extraits sonores, et retrace l'histoire de l'immigration en France depuis la fin du XIXème siècle, comme on peut avoir un aperçu sur l'image 4 (Figura 4).

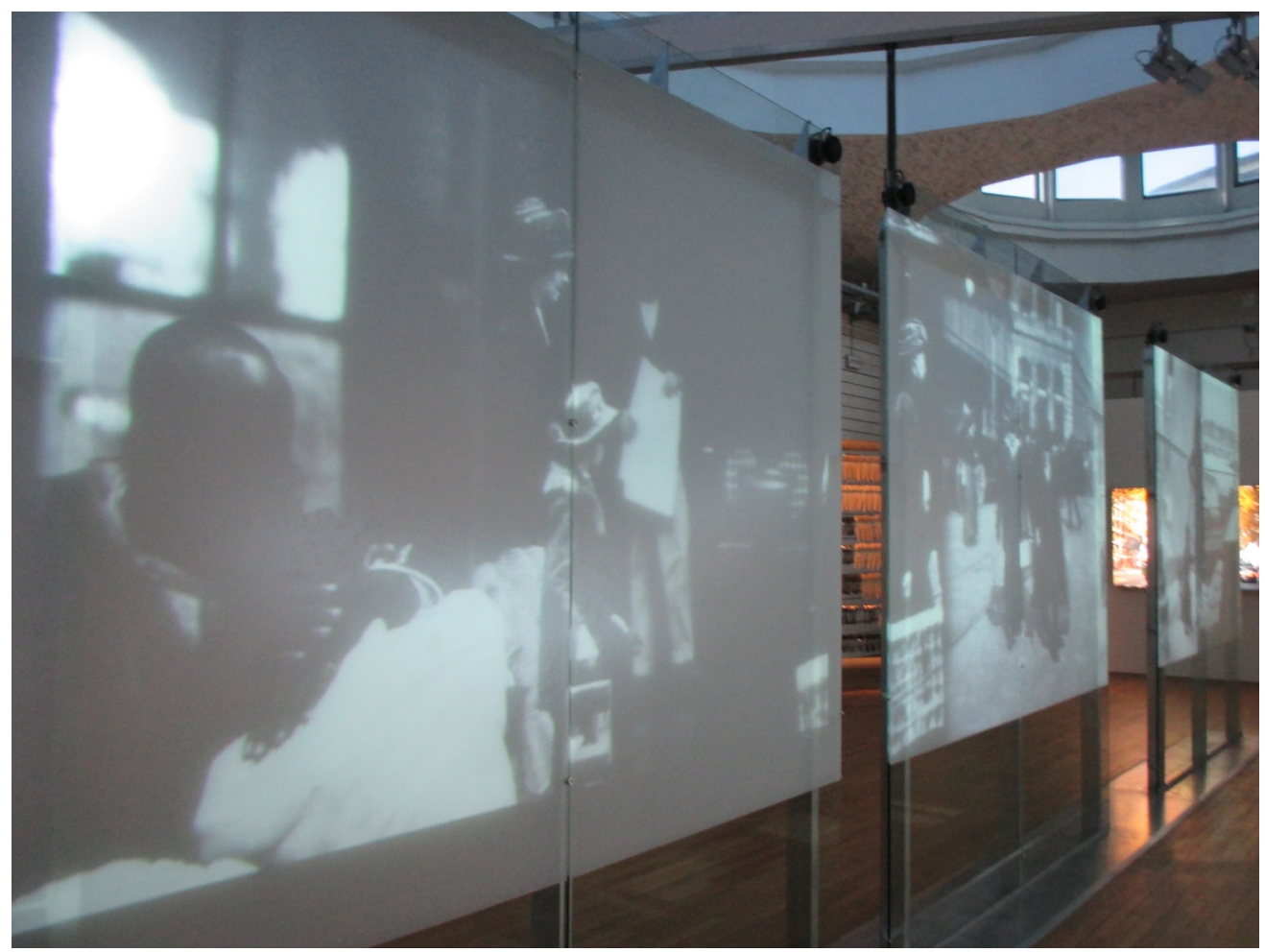

Figura 4 - Film sur l'immigration présenté à l'entrée de l'exposition Repères. Photographie de l'auteur, 2015.

Fabienne Muddu est chargée des collections photographiques du musée depuis 2007. Ayant travaillé à l'agence Magnum, en 2004, elle fut invitée à participer aux travaux de préfiguration de la collection photographique et à la préparation du film sur l'histoire de l'immigration.

Elle a effectué un travail de prospection photographique auprès d'agences comme Magnum pour retrouver des images susceptibles d'illustrer des projections et les tables rétroéclairées qui font partie de l'exposition permanente Repères. Ce travail de recherche d'images capables d'illustrer le discours du projet scientifique s'est accompagné également d'une prospection des photographies "dignes" d'entrer dans une collection ${ }^{33}$. Ainsi, l'acquisition des photographies avait lieu, lorsque possible, en pair avec le choix des illustrations. Parfois, l'acquisition des photographies ne se faisait pas immédiatement et dans ce cas le musée a eu recours aux reproductions et a dû
32. Le film de quarante minutes se trouve en ligne sur le site du musée: <http:// www.histoire-immigration. fr/histoire-de-l-immigration/ le-film $>$.

33. Il faut rappeler que le musée n'avait pas de collections préexistantes et donc le travail de constitution des collections a été initié en même temps que celui de la mise en place de l'exposition permanente Repères. 
34. Cette information a été donnée par la chargée des collections photographiques, Fabienne Muddu, lors d'un entretien en Mars 2015.

35. Voir l'installation de Barthélémy Toguo, Climbing Down (2004); site de l'artiste: <http://www. barthelemytoguo.com/ 2oeuvres/2installations/ climbing.html>, vérifié le 18 décembre 2015. payer des droits d'auteur aux agences photographiques détenant les droits des photographes.

C'est le cas de la photo de Patrick Zachmann "La procédure de régularisation exceptionnelle organisée sous un chapiteau par la Préfecture de Bobigny (1981)", qui a été initialement utilisée sur une des tables rétroéclairées de l'exposition permanente avant l'achat d'un tirage photographique d'époque à l'agence Magnum. Maintenant l'original de cette photographie est exposé pas très loin de sa reproduction. Comme actuellement la plus grande partie des images qui illustrent l'exposition Repères a été acquise, l'idée est donc de remplacer dans un futur proche les images empruntées ou reproduites par des images de la collection du musée ${ }^{34}$.

Parcours de l'exposition

L'exposition permanente Repères peut être divisée en trois grandes parties thématiques. Une première partie où l'expérience migratoire est relatée en faisant le lien entre histoire collective et histoires individuelles. Cette partie propose une analyse des raisons du départ, du choix de la France, du voyage, de la frontière, de la construction de l'étranger par le juridique et par le regard de l'autre. La deuxième partie aborde la question de l'habitat (l'exclusion et l'entre soi), le travail et le creuset. La dernière partie est centrée sur l'apport de cultures d'origines très diverses, autour de thèmes tels que la langue, les identités et les pratiques religieuses, la diversité des communautés immigrées et sur les multiples enrichissements apportés à la culture française par les cultures étrangères notamment dans les domaines de l'architecture, des arts plastiques, du cinéma, de la danse, de la littérature et de la musique.

Le parcours veut, ainsi, refléter l'itinéraire d'un immigré, bâti autour de quatre moments-clés:

1. le temps du voyage décrit à travers une frise d'images projetées sur des écrans, en regard des objets modestes que l'on emporte dans une valise.

2. les lieux de vie représentés par des photographies d'auteurs, accrochés sur des cimaises percées de fenêtres qui dessinent des rues et des cours et par une installation faite de plusieurs lits superposés ${ }^{35}$.

3. les sagas du travail, du sport et des luttes, dont les immigrés sont des acteurs essentiels-, racontées sur de grands tableaux numériques animés.

4. le concept d'intégration, "ferment de la diversité culturelle française", illustré dans les salons récréatifs ouverts sur la ville qui présentent des jeux interactifs, des films et des chansons, dans une ambiance de fête.

Ce parcours est ensuite sous-divisé en différentes parties: Émigrer; Face à l'Éfat; Terre d'accueil, France hostile; Ici et là-bas; Lieux de vie; Au travail; Enracinements; Sportifs; Diversité. Des photos, des objets présentés toujours dans des dioramas thématiques, des installations d'art, des registres audio et des documents d'archives illustrent ces différents thèmes et dialoguent entre eux, offrant ainsi au visiteur un questionnement de sa connaissance de l'immigration. 
Il faut décrire maintenant le parcours, en donnant une idée du contenu principal de chaque partie, pour que l'on puisse ensuite faire une analyse de la structure de l'exposition permanente

Description du parcours

Cette exposition se trouve au 2ème étage du Palais de la Porte Dorée dans un espace réaménagé pendant les travaux de restructuration du bâtiment.

Le parcours de l'exposition permanente s'ouvre sur un dispositif cartographique. Initialement attachés au plafond, trois groupes de quatre cartes géographiques géantes expliquent les différents mouvements migratoires vers la France, les lieux d'installation des immigrés au tournant du XXème siècle, dans les années 30, pendant les Trente Glorieuses et aujourd'hui. Selon les réalisateurs du projet, "dans l'ouverture se dévoile le projet d'une exposition. [...] Nous avons donc conçu une installation de trois ensembles de cartes géantes pour une entrée monumentale qui brosse les variations d'échelles des migrations dans l'espace et le temps, sur deux siècles. La France est resituée dans un espace mondialisé des migrations: au fil des décennies, l'origine géographique des migrants s'élargit des frontières au reste de l'Europe, puis à l'Afrique et au reste du monde" 36 .

Depuis 2014, ces cartes sont présentées sur un écran audiovisuel. Une chronologie sur les murs de l'escalier rappelle les moments forts concernant l'histoire de France et l'immigration. Elle accompagne le visiteur lorsqu'il remonte les escaliers qui mènent au 2ème étage. II s'agit d'une sorte de prologue qui permet de visualiser et de contextualiser l'immigration en France.

Ainsi, une fois dans l'exposition, on découvre des boîtes (avant colorées et depuis 2014 transparentes) qui contiennent plusieurs objets : valise, châle, statuette, vinyle, photos, etc. Chaque boîte contient aussi des extraits de témoignages des personnes qui ont donné les objets. Ces témoignages sont en vidéo dans certaines boites ou alors écrit dans d'autres. Ce sont des objets qui illustrent donc différents parcours et histoires d'immigrés originaires de différentes parties du monde.

La salle ne possède pas une illumination très forte et les images sont projetées sur des lames de verres. L'ambiance reste donc un peu obscure mais, depuis 2014, la salle est beaucoup plus claire suite aux critiques à ce manque d'illumination. L'impression ressentie est d'être dans un grenier où l'on garde des souvenirs. L'atmosphère est complétée par des extraits audio (témoignages, musique...). C'est comme si, dans cette première partie de l'exposition, qui s'appelle Émigrer, le visiteur était invité à se plonger dans la mémoire des immigrés. C'est une invitation à partager des mémoires et des histoires, parfois douloureuses, de personnes d'origines très diverses mais qui ont en commun l'expérience migratoire. On retrouve ici la volonté de mettre un visage à l'immigré qui normalement n'est jamais vraiment perçu comme un individu et plutôt vu comme l'autre, l'étranger lou étrange étranger comme dans le poème de Jacques Prévert) ${ }^{37}$.
36. Cf. Jean-Luc Marty (2008).

37. Cf. Jacques Prévert (2000). 
Plusieurs photographies sur le thème du voyage, l'arrivée ou le départ, ont été choisies par leur contenu marquant. Elles sont évocatrices des épreuves vécues par les immigrés. A nouveau, le choix des images exposées exprime la recherche d'un équilibre entre images inédites et photographies considérées comme incontournables ou emblématiques pour illustrer le thème de l'immigration. On retrouve, ainsi, des photographes fameux tels que Robert Capa (Réfugiés espagnols pendant leur transfert au camp du Barcarès, mars 1939) ou David Seymour (Plus de 400.000 réfugiés ont quitté l'Espagne lors de la chute du régime républicain, Le Perthus, février 1939).

Une table de repères chronologiques, comme on peut voir sur l'image 5 (Figura 5), présentant un axe du temps sur l'histoire de l'immigration en France offre des informations complémentaires sur les différentes vagues d'immigrés et pousse le visiteur à découvrir un peu plus cette histoire de l'immigration.

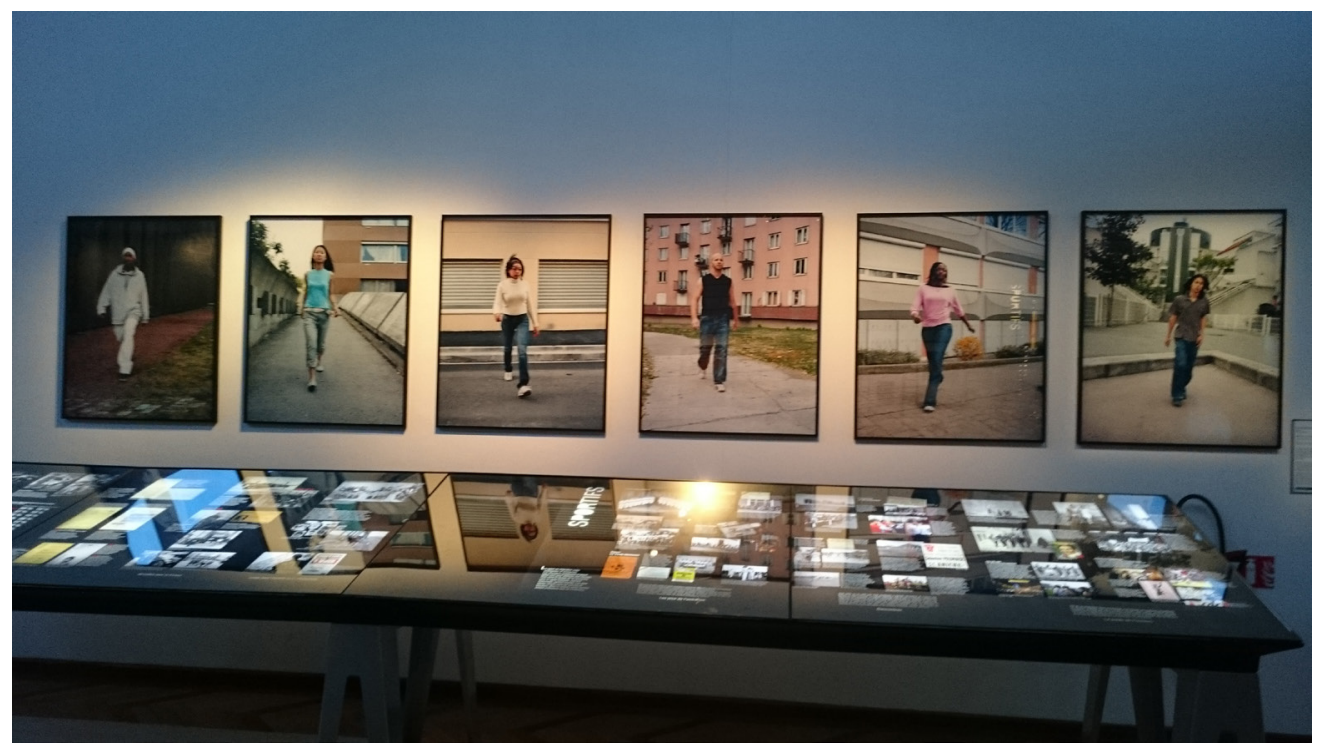

Figura 5 - Table de repères chronologiques et série photographique de Darzacq. Le nom de l'exposition permanente Repères vient d'ailleurs de ces tables de repères. Photographie de l'auteur, 2015.

Deux œuvres d'art qui portent sur la notion de mouvements migratoires complètent cette première partie. L'œuvre de Karim Kal, Images d'Alger 2002 (2003), consiste en quatre photographies numériques prises d'Alger vers la Méditerranée. Par ces photos, Karim Kal interroge la notion de mouvements migratoires (la nécessité et le désir). Les photos sont imprimées sur des posters et laissées sur un support en bois au sol. Dans un premier moment, le visiteur est attiré par ces posters qu'il peut prendre et garder, et c'est seulement après qu'il commence à se demander d'où ont été prises ces photos sur les posters.

Les images projetées sur les quatre lames écrans forment une immense fresque narrative des conditions de départ des immigrés, de leur voyage et du passage de la frontière jusqu'à leur arrivée en France. Des photographies de 
différentes vagues migratoires se succèdent et glissent sous les yeux du visiteur. C'est une œuvre plastique attirante par sa dynamique et le visiteur est attiré par cette succession d'images.

Deux fictions ponctuent le déroulement des images : une séquence consacrée aux lieux de passage aujourd'hui, une autre aux bagages. Pour cette dernière, huit personnes ont accepté de refaire" devant une caméra leurs valises comme elles les avaient préparées lors de leur départ pour la France. La séquence se termine par l'énumération du contenu de leurs bagages. Selon les réalisateurs, "[...] un très grand dispositif - des lames de verre de 12 mètres de long sur 2 mètres de hauteur - projette le visiteur au coeur de la problématique et donne chair aux flux démographiques cartographiés. Pour mettre en scène l'expérience migratoire sur ces deux siècles, nous avons voulu renouveler un point de vue, interroger des photographies d'archives et les réinscrire dans une vision contemporaine. Ces projections d'images proposent une véritable œuvre plastique produite à partir d'un montage d'images anciennes fixes et de séquences vidéo tournées pour l'occasion, instaurant de la sorte un dialogue, comme une variation poétique, autour des figures récurrentes des migrations: frontières, bagages, transports..." 38 .

La deuxième partie - Face à l'État; Terre d'accueil, France hostile expose une série de dessins, caricatures et charges parus dans des journaux, ainsi que des affiches et photos questionnant les politiques d'immigration.

Une série d'affiches avec le mot "Urgent" en lettres capitales attire l'attention du visiteur qui se trouve devant l'œuvre de Ghazel, artiste née à Téhéran et qui utilise son expérience personnelle d'immigrée pour créer son œuvre. En 1986, Ghazel quitte I'Iran pour venir étudier en France et, en 1997, alors qu'elle est installée à Montpellier, elle reçoit une lettre d'expulsion lui signifiant que sa carte de séjour ne sera pas renouvelée. Selon l'artiste: "Pour la première fois je me sentais sans papiers, clandestine". C'est ainsi, qu'elle décide de créer le projet artistique Urgent où elle met des annonces pour un mariage blanc. Ce sont plusieurs affiches déclinées en plusieurs versions sur le même thème. En 2002, date à laquelle elle reçoit une carte de résidence pour dix ans, elle renverse alors les données et propose à son tour un mariage à un clandestin afin qu'il puisse obtenir une carte de séjour. Si son travail puise dans les registres de l'humour, de la dérision et du détournement, il dépasse aussi la propre expérience de l'artiste et confère à l'œuvre une portée universelle. Ici, l'œuvre d'art apporte une critique souple mais très directe aux situations vécues par les "sans-papiers". Le mariage pour obtenir un visa étant très présent dans la réalité des immigrants "sans-papier".

Dans cette partie on trouve à nouveau une table rétroéclairée avec plus d'informations pour que le visiteur puisse approfondir le thème s'il le désire. On voit un box avec une vitrine contenant des marionnettes du XIXème siècle représentant différents personnages "étrangers" tels que l'arabe, la bretonne, le nègre, la femme voilée, la gitane, le brigand, Nelusko ${ }^{39}$ et l'usurier. Ils montrent la crainte de l'autre qui faisait partie de l'imaginaire de l'époque ${ }^{40}$. Ici on retrouve le registre historique
38. Cf. Jean-Luc Marty (2008).

39. Esclave africain, personnage de l'opéra L'Africaine (1865) de Giacomo Meyerbeer sur un livret d'Eugène Scribe.

40. Ces marionnettes sont très caricaturales et représentent ces personnages avec des traits très stéréotypés. 
41. Cf. CNHI, Guide de l'exposition permanente (2009).

42. Cf. CNHI, Guide de l'exposition permanente (2009). où l'objet témoin apparait comme essentiel pour comprendre l'imaginaire français du XIXème siècle envers l'étranger.

La troisième partie - Ici et là-bas; Lieux de vie - présente plusieurs photos de communautés immigrés dans leurs lieux de vie. L'idée est de montrer les différents types de logements d'immigrés au long du temps, des meublés et garnis de la fin du XIXème siècle jusqu'aux cités des années 1970. Plusieurs photos documentent les quartiers d'immigrés, la "zone", le logement patronal, les bidonvilles, les foyers Sonacotra et finalement les cités.

L'installation de Barthélémy Toguo, Climbing down, comprend six lits superposés en bois, quatre échelles et quarante sacs multicolores. Lorsque le visiteur découvre cette énorme installation, entourée de photos comme on peut observer sur l'image 6 (Figura 6 ), il est étonné et surpris. L'œuvre suggère les tensions qui peuvent exister entre l'espace public du foyer, l'environnement partagé par tous, et la sphère privée où chacun tente de reconstruire son univers propre, balisé par des sacs et des valises renfermant les traces du domicile déserté. La frontière entre le personnel et le collectif, l'extérieur et l'intérieur, devient alors ténue ${ }^{41}$.

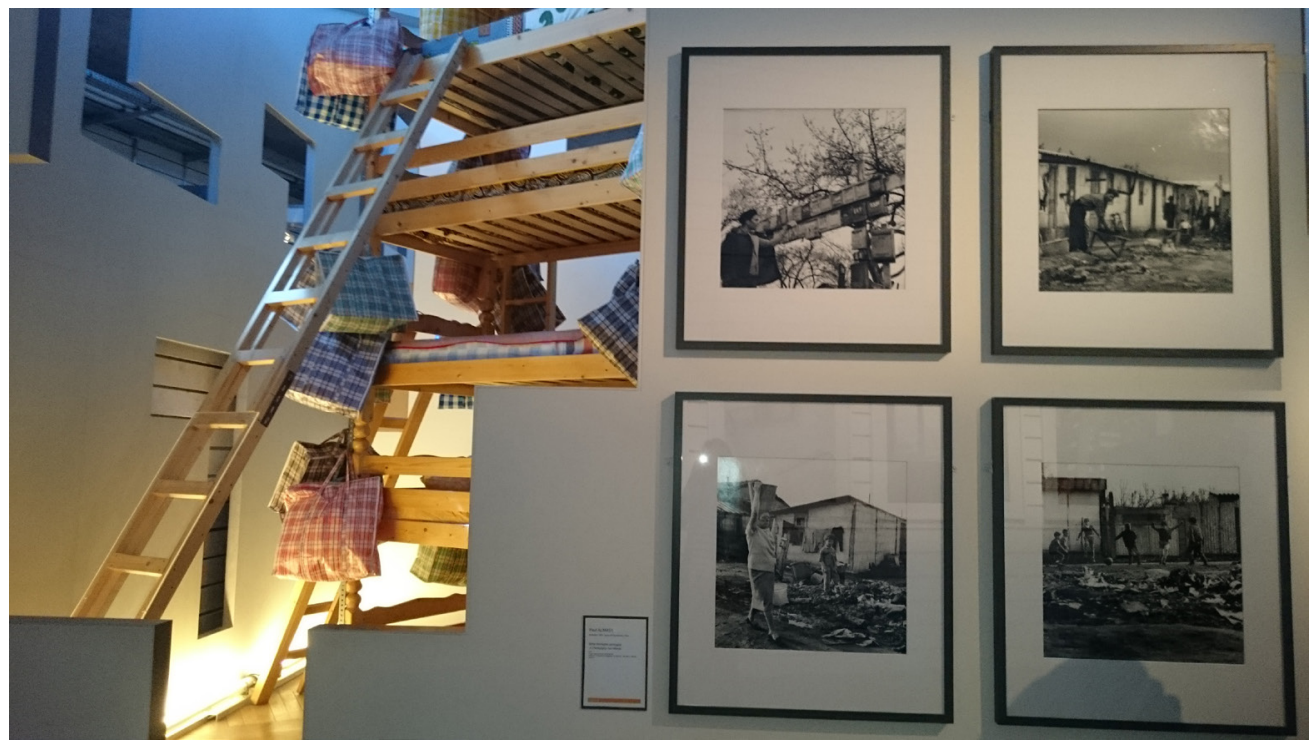

Figura 6 - Premier plan: Série photographies Immigrés portugais à Champigny-sur-Marne, Paul Almasy, 1963. Arrière plan: OEuvre de Barthélémy Toguo, Climbing Down, 2004. Photographie de l'auteur, 2015.

Une autre œuvre d'art qui attire beaucoup l'attention du visiteur est une série de photos de voitures appelées Les voitures cathédrales (2004). Cette œuvre de Thomas Mailaender se réfère au terme générique inventé par les dockers du port de Marseille et qui désigne ces véhicules transportant, par bateaux, des tonnes de marchandises de Marseille vers le Maghreb. Selon Mailaender, "en constant va-etvient entre les rives de la Méditerranée, ces containers roulants sont une matérialisation évidente du concept de frontière et des frottements culturels qui en découlent" $"$ 2. 
Déjà, Le Grand Ensemble, réalisé par Mathieu Pernot entre 2002 et 2006, fait référence au nom donné aux quartiers d'habitat social construits à la périphérie des grandes villes françaises à partir des années 1950 et destinés à répondre à la crise du logement et à la poussée démographique ${ }^{43}$. L'œuvre se présente sous la forme d'un corpus composé de trois ensembles d'images qui viennent frôler les temporalités et interroger l'histoire. Une photo grand format noir et blanc dévoile l'implosion d'un immeuble à Mantes-la-Jolie ${ }^{44}$ : Cette destruction violente, qui fait tout disparaître, pulvérise le passé, la mémoire du quartier et les promesses du lieu.

Mathieu Pernot associe à cette photographie d'implosion, Le Meilleur des Mondes, un ensemble de cartes postales, éditées entre 1950 et 1980, qu'il a collectionnées, reproduites et agrandies. Ces cartes postales, conçues pour la plupart en noir et blanc puis colorisées, nous rappellent que ces grands ensembles étaient alors des emblèmes de modernité urbaine et de progrès social, l'habitat social à son apogée. Ces bâtiments, qui aujourd'hui sont démolis et effacés du tissu urbain à cause de leur laideur et décadence, ont été à l'époque de leur construction une promesse de confort et de vie meilleure pour beaucoup de familles d'immigrés ${ }^{45}$. Mathieu Pernot crée une œuvre en palimpseste où l'association d'images autorise cet "aller-retour entre les rêves d'hier et les désillusions d'aujourd'hui". Mais, constate le photographe, "entre l'utopie de départ et la mise à mort finale, c'est comme s'il n'y avait rien eu"46.

La quatrième partie Au travail montre les étrangers dans leurs lieux de travail. Les sagas du travail dont les immigrés sont des acteurs essentiels, sont racontées sur de grands tableaux numériques animés. Plusieurs photos et vidéos des immigrés dans leurs lieux de travail à différentes périodes sont projetées sur un premier écran. Ensuite, sur un autre écran, on voit des photos et vidéos de manifestations de travailleurs immigrés.

Dans cette partie, on trouve à nouveau des œuvres d'art sur la question du travail immigré. Rajak Ohanian nous propose Le portrait d'une PME47 (1999) qui consiste en 32 portraits photographiques, en pied et grandeur nature, de l'ensemble du personnel d'une entreprise d'impression sur tissu de la région lyonnaise. Chaque employé a fait face à l'objectif avec pour toile de fond le tissu fait par lui-même le jour où la photo a été prise. "Chaque personne est un trentedeuxième du tissu qui sert de toile de fond à chaque photo. Avec ces prises de vues, j'ai voulu mettre en valeur la dignité de chacune de ces personnes"48.

Déià les œuvres de Chéri Samba, Assedic-ANPE (1982) et Paris est propre (1989) sont bien plus critiques et directes. Ces tableaux montrent la radicalité plastique et narrative qui marque les œuvres de l'artiste. Des phrases comme : "Grâce à nous les immigrés qui n'aimons pas voir les urines et les crottes des chiens", ou encore, "Sans nous, cette ville serait peutêtre la scorie de crottes" sont directes et tranchantes.

La cinquième partie Enracinements; Sportifs propose une discussion sur l'intégration des immigrés dans la société française et, en particulier, l'intégration
43. Avec la poussée démographique de cette période beaucoup de bidonvilles sont apparus dans les régions périphériques de Paris et ces quartiers d'habitat social étaient la grande promesse de résolution des problèmes d'habitation.

44. Quartier d'habitat social périphérique au nord de Paris.

45. Le mot " cité " est utilisé pour se référer à ces quartiers d'habitat social qui regroupent beaucoup d'immigrés ou de français issus de l'immigration. Malheureusement, le mot possède une connotation négative puisqu'il fait souvent référence à des lieux de pauvreté et de violence. Voir le film de Mathieu Kassovitz La haine (1995) qui montre bien l'ambiance de ces cités.

46. Cf. CNHI, Guide de l'exposition permanente (2009).

47. Le portrait d'une PME (1999), 32 épreuves sur papier baryté Ilford FB $1 \mathrm{~K}$ contrecollées sur dibon 2 $\mathrm{mm}$.

48. Cf. CNHI, Guide de l'exposition permanente (2009). 
49. On retrouve aussi une reproduction de La Joconde de Léonard de Vinci qui est devenu un symbole et qui attire des milliards de visiteurs au musée du Louvre pour la voir et prendre des photos. dans le domaine sportif. Là, à nouveau, plusieurs documents historiques tels que photos, demandes de naturalisation et décrets de naturalisation, donnent un témoignage plus concret de cette intégration ou volonté d'intégration à la société française. L'accueil des étrangers, l'école, le quotidien, la langue, l'accès à la nationalité, l'engagement des étrangers pendant les périodes de guerre, ainsi que, les questions d'égalité et de citoyenneté sont abordés dans cette partie de l'exposition, à partir, donc, de documents historiques, témoignages et histoires de vie.

Les parcours de vie d'Anatole Lewistsky et Boris Vildé sont pris en exemple. Ces deux immigrés d'origine russe sont arrivés en France respectivement en 1924 et 1933 et ont entamé une carrière d'ethnologue au Musée de l'Homme. Ils ont été naturalisés français dans les années 30 et ont fondé le Réseau du Musée de l'Homme après l'armistice de juin 1940. Ce Réseau, qui compte parmi les premières organisations de résistance en France, s'est accru et structuré rapidement avec l'aval de Paul Rivet, directeur du musée à l'époque, et a publié un journal clandestin appelé "Résistance", produit dans les sous-sols du musée. Son premier numéro sortit le 15 décembre 1940 et Boris Vildé en rédigea intégralement la une. En relation permanente avec la France libre, ils renseignaient également les forces alliées ce qui permettaient de libérer des prisonniers. Ils seront arrêtés et exécutés par la Gestapo en 1941. Leur histoire de vie et le souvenir de leurs actions restent toujours un exemple de "lutte partagée".

Dans la dernière partie de l'exposition "Diversité" on retrouve les apports et échanges culturels venant de l'étranger. Là il s'agit de montrer les emprunts, les adoptions et les métissages entre les cultures étrangères et la culture du pays d'accueil. Ainsi, le visiteur trouve une série de vidéos sur le thème principal de l'immigration, comme par exemple des extraits de films sur l'immigration ou encore des jeux où l'on doit deviner quelle est l'origine des mots sur l'écran.

Tout à la fin de l'exposition, se trouve l'espace Rencontre où plusieurs objets sont exposés. On peut regrouper ces objets en trois ensembles qui traitent des différents types d'adoptions et d'emprunts entre culture d'accueil et cultures immigrées.

Un premier ensemble regroupe des objets devenus typiquement français. Le visiteur découvre que plusieurs objets assimilés à des symboles français ${ }^{49}$ sont, en fait, le résultat d'emprunts ou d'adoptions venant de cultures étrangères. Un bon exemple en est l'accordéon dit musette. Au XIXème siècle, les cafés parisiens des quartiers populaires étaient animés par des auvergnats qui jouaient un instrument ressemblant à une cornemuse, surnommé de musette ou encore cabrette. Vers la fin du XIXème, la proximité sociale entre immigrés italiens et auvergnats provoque le remplacement de la cabrette par l'accordéon italien et c'est ainsi que de cette rencontre nait le musette, un genre musical considéré typiquement français.

Le deuxième ensemble traite des échanges culinaires et rappelle que les échanges de saveurs et produits alimentaires est aussi à la base de la dynamique des rapprochements culturels entre immigrés et culture nationale. Un exemple très 
parlant est le couscous, un des symboles culinaires des populations du Maghreb qui s'est rapidement répandu autour de la Méditerranée, à la faveur des voyages, du commerce et des migrations. Ainsi, le visiteur se retrouve face à une série d'objets liés aux pratiques culinaires dont un couscoussier.

Le troisième ensemble réunit plusieurs objets typiques d'autres cultures qui sont devenus familiers dans le pays d'accueil, comme par exemple, les lanternes chinoises. Cette partie est dénommée "Quand les objets de là-bas deviennent des objets familiers ici". Cette mise en scène de ces différents objets par l'équipe du musée est très réussie et propose une approche doublement subjective et interrogatrice du phénomène d'intégration.

Dans chaque partie de l'exposition permanente, il y a une partie "objet témoin" et une partie "œuvre d'art contemporain". Ces deux parties dialoguent entre elles et offrent au visiteur un panorama plus approfondi de la question. Le visiteur est appelé à faire partie d'un dialogue entre histoire, témoignages et expérience subjective et contemporanéité du sujet traité.

On retrouve cette dualité et ce dialogue dans toutes les expositions de la $\mathrm{CNHI}$, permanente ou temporaires. Les documents historiques et les objets témoins constituent le bastion de la collection du musée en apportant un regard plus objectif et direct sur les questions liées à l'immigration. Les œuvres d'art contemporain apportent un regard plus subjectif et interrogateur sur les différents processus du phénomène migratoire. La question de l'archive en histoire et l'importance de celleci dans la création contemporaine enrichissent ce dialogue encore plus riche. Aujourd'hui, le travail sur archives n'est plus une exclusivité des historiens ou des chercheurs, l'artiste lui aussi utilise les archives pour construire sa réflexion et son œuvre. Le processus créatif s'imprègne ainsi des démarches et des codes de l'archive ${ }^{50}$.

Le mélange objets témoins et œuvres d'art rend l'exposition plus intéressante et moins fatigante pour le visiteur. Cette volonté d'une mise en scène plus attirante pour le visiteur est très présente dans le projet scientifique du musée et dans le discours des muséographes de l'exposition permanente, Lydia Elhadad et Pascal Payeur ${ }^{51}$. La CNHI se veut un musée d'histoire contemporain, hors des cadres traditionnels et ringards des musées d'histoire. La mise en exposition reste une traduction spatiale et sensorielle, particulièrement visuelle, d'un discours et la matérialisation de ce dernier qui doit être suffisamment attirante pour capter l'attention du visiteur. Le musée qui autrefois était dédié à la conservation, à la présentation et à l'étude des collections devient musée spectacle ${ }^{52}$. Un musée multidisciplinaire et multi-communicationnel, interpellant diverses disciplines scientifiques, mais aussi différentes formes d'art ${ }^{53}$.

L'exposition permanente reste ciblée et structurée. Chaque partie de l'exposition rassemble objets témoins, archives photos, documents historiques, œuvres d'art et une table contient des informations complémentaires sur chaque partie pour que le visiteur puisse approfondir le sujet. Ces tables sont des repères
50. Un exemple intéressant est celui de la Tate Britain qui possède un espace dédié à la création artistique basée sur les archives de l'institution; voir <http:// www.tate.org.uk/whats-on/ tate-britain/display/bpspotlight-charlotte-mothchoreography-image>.

51. Voir Marie Poinsot (2007).

52. Aujourd'hui les musées se voient de plus en plus dans la situation délicate de devoir toujours attirer de plus en plus de visiteurs. Le contexte économique actuel lié à d'autres contraintes a poussé les musées dans des logiques de plus en plus liées à l'industrie de l'entertainment pour trouver du financement. Augmenter le nombre de visiteurs devient un des principaux objectifs des musées et leur succès est aussi mesuré en fonction de l'affluence de public.

53. La CNHI propose aussi des spectacles vivants et musicaux dans sa programmation. 
54. La médiathèque de la Cité, spécialisée en ouvrages sur l'immigration, fonctionne alors comme cet espace d'information où les visiteurs plus curieux peuvent approfondir les thématiques abordées dans l'exposition permanente.

55. Cf. le site CNHI, rubrique guide de l'exposition.

56. Suite à des entretiens réalisés en Mars 2015 avec Isabelle Renard et Isabelle Muddu, chargées de collections, le projet scientifique est en train d'être revu par l'équipe du musée pour qu'il soit transformé et mis à jour. Il reste à savoir si ces modifications en cours entraineront un changement réel du discours du musée. qui constituent la colonne vertébrale chronologique du parcours. L'objectif principal de l'exposition Repères est de donner, comme le nom le dit déjà, des repères et des pistes pour que le visiteur puisse mieux comprendre l'histoire de l'immigration en France et développer sa propre réflexion sur le sujet.

Pourtant il semble y avoir un manque de profondeur. Des questions plus délicates liées au thème de l'immigration ne sont pas vraiment traitées, comme par exemple les politiques d'immigration actuelles en France ou les problèmes liés à l'intégration. En effet, l'exposition consiste en un panorama sur le thème de l'immigration en France avec des thématiques qui d'une certaine façon introduisent la question. En conséquence, pour que le visiteur puisse approfondir les points présentés dans l'exposition, il doit aller chercher plus $d^{\prime}$ informations ailleurs ${ }^{54}$. Ne présentant pas une vision critique et approfondie sur l'immigration en France, l'exposition permanente est loin d'être une unanimité. Depuis son ouverture, le musée a reçu de nombreuses critiques qui convergent toutes vers une demande d'approfondissement et d'élargissement des thématiques traitées (comme par exemple développer la contextualisation du bâtiment ou parler de la question coloniale et des politiques d'immigration) et d'avoir un discours plus critique face à l'intégration de ces immigrés dans la société française.

En 2014, avec la réouverture de l'exposition Repères et de la Galerie des dons, le musée a revendiqué "une refonte complète de son parcours et un nouvel accrochage des œuvres; ce sont 200 ans d'histoire de l'immigration en France qui s'offrent aux visiteurs" 55 . Pourtant, les modifications restent superficielles puisqu'elles ne concernent que la muséographie (illumination de l'exposition pour augmenter la clarté, accrochage de nouvelles photographies et d'œuvres d'art...) mais ni le contenu, ni le discours de l'exposition ${ }^{56}$.

Conclusion

Si on dresse un bilan sur les sept premières années d'existence de la $\mathrm{CNHI}$, on retrouve un musée qui certainement n'a cessé de se transformer mais qui a du mal à trouver son espace dans le paysage muséal français. Peut-être la crise identitaire du musée est-elle liée aux tensions et problèmes de la société française face à la question de "I'identité nationale". La démission de huit membres du conseil scientifique de la CNHI, en mai 2007, pour protester contre la création d'un Ministère de l'Immigration, de l'Intégration, de l'Identité nationale et du Codéveloppement, montre bien l'enjeu et les contradictions présentes dans ce processus de patrimonialisation de l'immigration en France.

Si l'acte de mise en patrimoine peut être une affaire d'Etat en France, comme dans le cas de la CNHI, il convient de s'interroger sur le discours de celleci pour définir ce qui constitue la nation. Est-ce que l'inscription de l'immigration dans la politique patrimoniale de l'Ełat mène à une prise en compte du caractère multiculturel de la société française et de l'identité française? 
La conception intégratrice de l'identité nationale, voire même assimilationniste, bloque toute conception de diversité culturelle. La communauté nationale quand elle se définit comme achevée en tant qu'unité dont l'immigré doit se rapprocher, s'oppose à l'image d'une nation inclusive qui accepterait justement les différentes communautés issues du phénomène migratoire.

En outre, il est important d'analyser la façon dont le musée dresse l'image des immigrés et le discours qui est véhiculé à travers l'exposition permanente Repères. La création d'une collection à partir de zéro a été un défi relevé par la $\mathrm{CNHI}$ qui a littéralement construit un patrimoine de l'immigration basé sur le caractère mémoriel des objets et des récits rassemblés par le musée : photos, vidéos, œuvres d'art, documents d'archives, objets divers donnés par des immigrés. II est certain que la $\mathrm{CNH}$ reste une tentative d'agir sur la société et ses représentations à travers cette reconnaissance patrimoniale. Toutefois, le musée ne semble pas trouver vraiment un discours pertinent sur le sujet. L'exposition permanente ne fait pas l'unanimité auprès du public et du milieu académique ${ }^{57}$ depuis son ouverture en 2007, et, en conséquence, il essaie de se réinventer grâce aux modifications récentes de l'exposition Repères et de la Galerie des dons lles deux ont été réouvertes au public en 2014 ). II reste à savoir si le musée arrivera à trouver sa voix et sa place dans le paysage muséal français.

\section{REFERÊNCIAS}

APPADURAI, Arjun. The Social Life of Things: Commodities in Cultural Perspective (edited volume). New York: Cambridge University Press, 1986.

BARBE, Nöel; CHAULIAC, Marina. Mémoire des immigrés, patrimoine de l'immigration. In: (Ed.). L'immigration aux frontières du patrimoine. Paris: Maison des Sciences de L'homme, 2014. p. 5-24. (Cahiers d'ethnologie de la France).

CITÉ NATIONALE DE L'HISTOIRE DE L'IMMIGRATION. Projet Scientifique et Culturel (CNHI). Paris: CNHI, 2005.

CITÉ NATIONALE DE L'HISTOIRE DE L'IMMIGRATION. Dossiers de presse de la Cité nationale de l'histoire de l'immigration (CNHI). Paris: CNHI, 2007.

CITÉ NATIONALE DE L'HISTOIRE DE L'IMMIGRATION. Guide de l'exposition permanente. Paris: Gallimard, 2009.

CITÉ NATIONALE DE L'HISTOIRE DE L'IMMIGRATION. Rubrique nouvelle campagne de communication. Disponible sur le site: <http://www.histoire-immigration.fr/la-cite/dernieresnouvelles/nouvelle-campagne-de-communication>, vérifié le 18 décembre 2015.

CITÉ NATIONALE DE L'HISTOIRE DE L'IMMIGRATION. Rubrique guide de l'exposition. Disponible sur le site: <http://www.histoire-immigration.fr/musee/guide-de-l-exposition>, vérifié le 18 décembre 2015.
57. Voir Michel Guérrin (2010) et Michael Kimmelman (2007). 
GENERIQUES. Faire connaître l'histoire et la mémoire de l'immigration. 2015. Disponible sur le site: <www.generiques.org>, vérifié le 23 novembre 2015.

GUERRIN, Michel. Le musée fantôme. Le Monde, Paris, 19 mars 2010. p. 1-1. Disponible sur le site: <http://www.lemonde.fr/societe/article/2010/03/19/le-musee-fantome_1321510_3224. html>, vérifié le 23 novembre 2015.

HOLLANDE, François. Discours d'inauguration du Musée de l'histoire de l'immigration. Paris: Présidence de la République française, 2014. Disponible sur le site: <http://www.elysee.fr/ declarations/article/discours-d-inauguration-du-musee-de-l-histoire-de-l-immigration>, vérifié le 23 novembre 2015 .

IMMIGRATION: la contre-offensive de Hollande. Le Monde.fr, Paris, 15 décembre 2014. p. 1-1. Disponible sur le site: <http://www.lemonde.fr/politique/article/2014/12/15/immigration-lacontre-offensive-de-hollande_4540644_823448.html>, vérifié le 15 décembre 2014.

JOLY, Marie-Hélène. Une collection en devenir- La place de la Cité nationale dans le paysage muséal français. Hommes \& Migrations, Paris, n. 1267, p.68-82, mai-juin 2007. Trimestriel. Disponible sur le site: <http://www.hommes-et-migrations.fr/index.php?id=4801>, vérifié le 23 novembre 2015.

KIMMELMAN, Michael. Ready or not, France opens museum on immigration. The New York Times, New York, 17 octobre 2007. Disponible sur le site: <http://www.nytimes. com/2007/10/17/arts/design/17abroad.html?_r=0>, vérifié le 23 novembre 2015.

L'ESTOILE, Benoit de. Le goût des autres: de l'exposition coloniale aux arts premiers. Paris: Flammarion, 2007.

MARTY, Jean-Luc. Cité de l'histoire de l'immigration: mémoire vivante de l'identité française. Géo découverte, Paris, n. 24 (Les plus beaux musées de Paris), 2008. Hors-série.

POINSOT, Marie. La Cité Nationale de l'Histoire de l'Immigration. Quels Publics?. Hommes E Migrations, Paris, oct. 2007. Hors-série.

PREVERT, Jacques. Etranges étrangers et autres poèmes. Paris: Folio, 2012.

TOUBON, Jacques. Mission de préfiguration du Centre de ressources de mémoire de l'immigration, rapport au Premier ministre. Paris: La Documentation française, 2004.

Artigo apresentado em 28/04/2015. Aprovado em 13/08/2015. 